\title{
26 Research Square \\ Parental phone use during mealtime with toddlers and the associations with feeding practices and shared family meals: a cross-sectional study
}

Frøydis Nordgård Vik ( $\sim$ froydis.n.vik@uia.no )

Universitetet i Agder Fakultet for Helse og Idrettsvitenskap https://orcid.org/0000-0001-7324-0070

Erik Grasaas

Universitetet i Agder Fakultet for Helse og Idrettsvitenskap

Maaike E. M. Polspoel

Universitetet i Agder

Margrehte Røed

Universitetet i Agder

Elisabet Rudjord Hillesund

Universitetet i Agder Fakultet for Helse og Idrettsvitenskap

Nina Cecilie Øverby

Universitetet i Agder Fakultet for Helse og Idrettsvitenskap

Research

Keywords: parental phone use, toddlers, family meals, meals, feeding practices, Norway

Posted Date: June 18th, 2020

DOl: https://doi.org/10.21203/rs.3.rs-35917/v1

License: (1) This work is licensed under a Creative Commons Attribution 4.0 International License.

Read Full License 


\section{Abstract}

\section{Background}

Positive parental feeding practices and a higher frequency of family meals are related to healthier child dietary habits. Parents play an essential role when it comes to the development of their child's eating habits. However, parents are increasingly distracted by their mobile phone during mealtime. The aim of this study was to describe the feeding practices and daily shared family meals among parents who use and do not use mobile phone during mealtime, and further to explore the associations between the use of mobile phone during mealtime and feeding practices and daily shared family meals, respectively.

Methods

Cross-sectional data from the Food4toddler study were used to explore the association between mobile use during meals and parental feeding practices including family meals. In 2017/2018 parents of toddlers were recruited through social media to participate in the study. In total 298 out of 404 who volunteered to participate, filled in a baseline questionnaire, including questions from the comprehensive feeding practices questionnaire (CFPQ), questions of frequency of family meals and use of mobile phone during meals.

Results

Herein, 4 out of 10 parents reported various levels of phone use (meal distraction) during mealtimes. Parental phone use was associated with lower use of positive parental feeding practices like modelling $(B=-1.05(95 \% \mathrm{Cl}-1.69 ;-0.41))$ and family food environment $(B=-0.77(95 \% \mathrm{Cl}-1.51 ;-0.03))$, and more use of negative parental feeding practices like emotional regulation $(B=0.73(95 \% \mathrm{Cl} 0.32 ; 1.14))$ and the use of pressure to eat $(B=1.22(95 \% \mathrm{Cl} 0.41 ; 2.03))$. Furthermore, parental phone use was associated with a lower frequency of daily family breakfast $(\mathrm{OR}=0.50(95 \% \mathrm{Cl} 0.31 ; 0.82))$ and dinner $(\mathrm{OR}=0.57(95 \% \mathrm{Cl}$ $0.35 ; 0.93)$ ).

\section{Conclusions}

Mobile phone use is common among parents during mealtimes, and findings indicate that parental phone use is associated with less healthy feeding practices and shared family meals. These findings highlight the importance of making parents aware of potential impacts of meal distractions.

\section{Introduction}

The importance of promoting healthy eating habits early in life cannot be stressed enough. The recent report from the WHO-UNICEF-Lancet commission: A future for the world's children? states that investing in young children's health, education, and development are fundamental for the individual's lifelong health and development, and even for their future children's health (1). This report also highlights the importance of good health and nutrition in the prenatal period and early years in order to lay the 
foundation for a healthy life course (1). An unhealthy diet early in life tend to endure into adulthood (2) and increases the risk of non-communicable diseases (NCD's) (3) and childhood obesity (4). Therefore, developing healthy eating habits as a child is essential for maintaining good health throughout life (5). There are also large societal economic benefits to be made if the population follow dietary guidelines (6).

Different determinants will influence the forming of the child's eating habits. At one year of age, the toddler's eating habits are mostly influenced by their parents i.e. the parents will decide what, when and how much the child eats $(7,8)$. Mealtimes serve as a setting for socialization around food and eating for the child together with their family (9). The feeding practices the parents use are important in developing eating habits for their children and they can promote or restrain a healthy diet (10). Parental feeding practices are defined by Shloim et al. (11) as specific goal-directed behaviors that parents use to directly influence the children's eating. Parental feeding practices can be positive or negative in forming eating habits among small children. An example of a negative feeding practice is if parents pressure their child to eat a particular food item (e.g. broccoli), it may lead to disliking of the actual food item (broccoli), while involving the child in food preparation (e.g. scrambled eggs) can be positive and increase the willingness to try new food (12).

According to Internet Growth Statistics (13), the usage of digital screens, e.g. computers, mobile phones and tablets have increased largely worldwide over the last decades. Everyone, including people in lower socio-economic groups, e.g. with a low salary, own a mobile phone, and it is increasingly considered a "must have" object for almost everyone; richer and poorer. With increasing screen use among the population, our behaviours change. It is common to see people walking outside using their phone, people at restaurants doing the same, even when they are together with other people.

A recent study found that distractions take up a large proportion (almost half) of the average family mealtime (14). Distractions at mealtimes (e.g. screens) have been shown to be associated with greater intake of unhealthy food (15). When mealtimes are characterized by disruptions, lack of attention to each other's moods and eating behaviors, and being away from the table, there is increased risk for poor dietary habits and a stressful eating environment $(16,17)$. During mealtimes with no distractions (e.g., no television (TV), phones, toys), preschoolers have been observed engaging in less fussy eating behaviors, whereas mealtimes with a distraction were characterized by more negative child behavior (18). There is limited research on implications of distractions on parent feeding practices. One study showed that mothers of infants ( $<6$ months) reported that $26 \%$ of the total feeding time was interrupted by technological use (TV, computer, smartphone, tablet) while $17 \%$ of total feedings was interrupted by nontechnological distractions (reading, housework) (19). Father availability and involvement in feeding practices have been studied in a recent paper and showed that fathers' absence at mealtimes was associated with more child distractions and less maternal feeding responsiveness (14).

Family meals are defined in different ways, depending on the number of family member who are attending (e.g. are one or both parents present?) or if the setting in which the meal takes place is included (e.g. is the meal at the table or in front of the TV?). Verhage et al uses the definition: The family meal can 
be seen as a social moment of the day during which food is eaten together with at least one family member (20). Frequency of family meals are often linked to childhood obesity. A meta-analytic review of 17 studies including more than 180,000 children and adolescents indicated that regularly sharing meals as a family reduced the odds for child overweight by $12 \%$ and increased the odds for eating healthy foods by $24 \%$ (21). But frequency of family mealtimes are also related to healthier eating behaviors in children (10).

We hypothesized that parental use of mobile phone during mealtime would be associated with less healthy feeding practices and lower frequency of daily shared family meals. Thus, the objective of this paper was to describe the parental feeding practice and frequency of daily shared family meals among parents who use and do not use mobile phone during mealtime; and further to explore the association between the use of mobile phone during mealtime, feeding practice and daily shared family meals.

\section{Methods}

The recommendations of STROBE-nut (Strengthening the Reporting of Observational Studies in Epidemiology-Nutritional Epidemiology) were followed (see Additional File 1).

Study design

Food4toddlers is originally a randomized controlled trial evaluating the effect of a digital dietary intervention targeting parents of 1 -year olds (22). The current paper reports on cross-sectional baseline data from this study.

Sample and procedure

In 2017 we recruited parents of toddlers. They were recruited through social media posts on Facebook. Parents voluntarily notified that they wanted to participate after reading written information about the study. In total 404 participants volunteered to participate initially, however of these, only 298 participants filled in the baseline questionnaire. Data from these 298 participants were included in the present study. The study was approved by the Norwegian Centre for Research Data, and by the Faculty ethics committee and has been conducted in line with the Helsinki Declaration of 1985, revised 2008.

Instruments

The baseline questionnaire included questions of parental and child characteristics like age and educational level, further there were questions regarding use of mobile devices, food frequency questions of child diet and parental diet, frequency of family meals, feeding practices and other intervention specific questions. In the current study the variables use of mobile device during mealtime (independent variables) and feeding practices and shared family meal frequency (dependent variables) are presented. In addition to parent and child characteristics. 
The use of mobile phone during mealtime, was reported like this: To what extent do you agree with the following statement: I often check my phone during meals. The participants were given the following response alternatives: Disagree, slightly disagree, neither agree or disagree, slightly agree and agree. We dichotomized this variable with the intention to differentiate between those who do not use mobile phones during meals at all and those who do. The participants who filled in "disagree" were defined as the "No phone use during meal" group, while the participants who filled in "slightly disagree, neither agree or disagree, slightly agree and agree" were defined as the "phone use during meal" group.

To assess feeding practices, we used the well-known and validated, Comprehensive Feeding Practices Questionnaire (CFPQ) (23). This questionnaire covers 12 dimensions of parental feeding practices. Because the participants of this study were parents of infants, following nine items were considered relevant: Child control (The child is allowed to control his/her own eating behaviours), Emotion regulation (The parents use food to regulate the child's emotional states), Balance and variety (Parents encourage well-balanced food intake), Environment (Availability of healthy food at home), Food as reward (Use of food to reward child behavior), Modeling (Parents demonstrate healthy eating behavior), Monitoring (Parents keep track of child's intake of less healthy food), Pressure (Used by parents to make child eat more), Restriction for health and weight control (Parents control the child's food intake with the purpose of limiting less healthy foods and sweets and to decrease or maintain the child's weight). The reliability of the ten feeding practices dimensions was evaluated using Cronbach alpha. All scores except Child control and Food as a reward $(a=0.3)$ showed acceptable reliability $(a=0.5-0.8)$ (detailed data not shown). The CFPQ items were previously translated from English into Norwegian in another project and a random sample of 10 items were back-translated into English (24). The quality of the translation was considered very good and this translation was therefore used in this project.

To assess the frequency of shared family meals, following questions were posed: How often does your child have the following meals together with their family? Herein, meals with family included parents that were married, living in cohabitant families, single or separated / divorced. The participants could choose from the following response alternatives: Never, 1, 2, 3, 4, 5, 6 and 7 times per week for breakfast, lunch, and dinner, respectively. Later, the weekly food frequency was divided into having every specific family meal, every day or not.

Parental age was assessed in years, child age in months. Participants reported their own and the other parent's highest level of education (primary school or less, primary schools plus one year of further education, high school, vocational school, upper secondary school or less, college/university ( $\leq 4$ years), college/university ( $>4$ years), other, don't know). These responses were dichotomized into having no university/college education or having university college education and used as a proxy for socioeconomic status (SES). Ethnicity of the parents were assessed as native if they were born in Norway or non-native if they were born elsewhere.

\section{Statistical analysis}


The statistical analyses were conducted using IBM SPSS Statistics for Windows, Version 25.0 (IBM Corp., Armonk, NY). Demographic data were described using descriptive measures. Continuous variables were described with mean and standard deviation, and categorical variables with frequencies and percentages. For the dependent study variable feeding practice, 9 out of 10 sub-scales had skewness values of \pm 1.3 and kurtosis values of \pm 1.6 , which indicated that these variables are approximately normally distributed. Thus, linear regression analyses were conducted between the dependent variables (feeding practices) on the independent dichotomy variable (phone use / no phone use), and then controlled for SES, ethnicity and age using multiple regression analysis. Binary logistic regression analyses were conducted to calculate odds ratios on the relationship between the dependent dichotomy variable shared family meals and the independent dichotomy variable (phone use / no phone use) controlling for SES, ethnicity and age. Due to the homogeneity of the sample in terms of gender ( $98 \%$ were mothers), adjusting for this variable was not considered relevant. P-values $<0.05$ were considered statistically significant and all tests were two-sided.

\section{Results}

\section{Participants}

The total sample included 298 parents with a mean age of 32.3 (SD \pm 4.2$)$ years and their children's age were 10.9 months (SD \pm 0.1 ). Table 1 presents the descriptive characteristics of the total sample. A great majority of the sample were mothers (98.7\%) and consisted of parents who were either married $(50.7 \%)$, lived in cohabitant families $(48.3 \%)$ or who were single $(0.7 \%)$ or divorced / separated $(0.3 \%)$ at the time of the study. The participants reported primarily a high education level, herein $53.7 \%$ reported more than 4 years of University level. Further, about 4 out of 10 parents reported using a mobile phone during mealtime with their children. Most parents were born in Norway, i.e. native. 
Table 1

Descriptive statistics of the sample $(n=298)$

\begin{tabular}{|cll|}
\hline Characteristics & Values & $\mathbf{N}(\%)$ \\
\hline Relationship to child, $N(\%)$ & Mother & $294(98.7)$ \\
\hline \multirow{2}{*}{ Education level, $N(\%)$} & Father & $4(1.3)$ \\
& Lower secondary school or less & $3(1.0)$ \\
\cline { 2 - 3 } & Upper secondary school & $23(7.7)$ \\
\cline { 2 - 3 } & College/University ( $\leq 4$ years) & $101(33.9)$ \\
\cline { 2 - 3 } & College/University (>4 years) & $160(53.7)$ \\
\cline { 2 - 3 } & Other & $11(3.7)$ \\
\hline Marital status, $N(\%)$ & Married & $151(50.7)$ \\
\cline { 2 - 3 } & Cohabitant & $144(48.3)$ \\
\cline { 2 - 3 } & Single & $2(0.7)$ \\
\cline { 2 - 3 } & Divorced/separated & $1(0.3)$ \\
\hline Ethnicity, $N(\%)$ & Native & $257(86.2)$ \\
& Non-native & $41(13.8)$ \\
\hline Child's gender, $N(\%)$ & Female & $134(45)$ \\
& Male & $164(55)$ \\
\hline
\end{tabular}

Descriptive data of the study variables: Phone use, Feeding practices and Shared Family Meals.

A total of 181 of the participants $(60.7 \%)$ reported no use of mobile phone during mealtime, wherein the remaining 117 participants $(39.3 \%)$ reported a various level of phone use during mealtime. The largest difference in mean for feeding practice was shown for the subscale pressure, where the group using phone during mealtime reported a mean score of 7.1 (SD \pm 3.2 ) compared to $5.8(S D \pm 3.6)$ the group with no phone use. Higher frequency of all types of shared family meals were reported by the group with no phone use compared to the group with phone use during mealtime (see Table 2). The highest frequency of shared family meals was dinner for both groups ( $71.3 \%$ and $57.3 \%$, respectively). 
Table 2

Characteristics of feeding practices and daily shared family meals stratified by phone use during mealtime.

\begin{tabular}{|c|c|c|}
\hline Dependent study variables & $\begin{array}{l}\text { Phone use } \\
N=117 \\
\text { (mean/SD) }\end{array}$ & $\begin{array}{l}\text { No phone use } \\
N=181 \\
\text { (mean/SD) }\end{array}$ \\
\hline $\begin{array}{l}\text { Feeding practice } \\
\text { Child control }\end{array}$ & $6.66(2.88)$ & $6.25(2.83)$ \\
\hline Emotional regulation & $3.74(1.70)$ & $2.98(1.73)$ \\
\hline Balance and variety & $14.07(1.90)$ & $14.44(1.80)$ \\
\hline Environment & $11.85(3.15)$ & $12.60(3.10)$ \\
\hline Food as reward & $1.31(1.69)$ & $1.18(1.53)$ \\
\hline Modelling & $12.54(2.91)$ & $13.56(2.59)$ \\
\hline Monitoring & $14.83(2.19)$ & $14.39(3.15)$ \\
\hline Pressure & $7.14(3.20)$ & $5.82(3.58)$ \\
\hline Restriction for Health & $5.78(3.22)$ & $5.65(3.21)$ \\
\hline Restriction for Weight & $6.20(3.88)$ & $6.22(4.34)$ \\
\hline Family meals & $58(49.6)$ & $121(66.9)$ \\
\hline Breakfast, $N(\%)$ & $34(29.1)$ & $72(39.8)$ \\
\hline Lunch, $N(\%)$ & $67(57.3)$ & $129(71.3)$ \\
\hline Dinner, $N(\%)$ & & \\
\hline
\end{tabular}

Associations between phone use during mealtime on feeding practice.

Phone use during mealtime was a significant predictor for the following feeding practice subscales: emotional regulation ( $95 \% \mathrm{Cl}[0.32 ; 1.14])$, environment (95\% Cl [-1.51; -0.03$])$, modeling (95\% Cl $[-1.69$; $-0.41])$ and pressure $(95 \% \mathrm{Cl}[0.41 ; 2.03])$ (Table 3$)$. After adjusting for SES, ethnicity and age, these respective associations remained significant with a $p$ value $<0.05$. 
Table 3

Associations between phone use during mealtime on feeding practice subscales adjusted for SES and age.

\begin{tabular}{|llll|}
\hline Feeding practices & $\mathbf{B}$ & $\mathbf{9 5 \%} \mathbf{C l}$ & P value \\
\hline Child control & 0.38 & $-0.29 ; 1.05$ & 0.27 \\
\hline Emotional regulation & 0.73 & $0.32 ; 1.14$ & $<0.001$ \\
\hline Balance and variety & -0.37 & $-0.80 ; 0.07$ & 0.09 \\
\hline Environment & -0.77 & $-1.51 ;-0.03$ & $<0.05$ \\
\hline Food as reward & 0.13 & $-0.25 ; 0.50$ & 0.51 \\
\hline Modeling & -1.05 & $-1.69 ;-0.41$ & $<0.001$ \\
\hline Monitoring & 0.49 & $-0.17 ; 1.15$ & 0.14 \\
\hline Pressure & 1.22 & $0.41 ; 2.03$ & $<0.01$ \\
\hline Restriction (Health) & 0.13 & $-0.64 ; 0.89$ & 0.74 \\
\hline Restriction (Weight) & -0.06 & $-1.04 ; 0.90$ & 0.90 \\
\hline B: Beta Cl: Confidence Interval SES: socio-economic status \\
\hline
\end{tabular}

Multiple regression analyses were used

Associations between phone use on shared family meals.

There was a $50 \%$ lower odds of breakfast $(95 \% \mathrm{Cl}[0.31 ; 0.82])$ and a $43 \%$ lower odds of dinner $(95 \% \mathrm{Cl}$ $[0.35 ; 0.93])$ as daily shared family meals among the group using mobile phone compared to the group with no phone use during mealtime.

Table 4

Associations between phone use on shared family meals adjusted for SES, ethnicity, and age.

\begin{tabular}{|lccc|}
\hline Shared family meals & OR & $95 \%$ Cl & P value \\
\hline $\begin{array}{l}\text { Breakfast } \\
\text { (phone use vs no phone use) }\end{array}$ & 0.50 & $0.31 ; 0.82$ & $<0.01$ \\
\hline $\begin{array}{l}\text { Lunch } \\
\text { (phone use vs no phone use) }\end{array}$ & 0.63 & $0.38 ; 1.05$ & 0.08 \\
\hline $\begin{array}{l}\text { Dinner } \\
\text { (phone use vs no phone use) }\end{array}$ & 0.57 & $0.35 ; 0.93$ & $<0.05$ \\
\hline \begin{tabular}{l} 
OR: Odds Ratio Cl: Confidence Interval SES: socio-economic status \\
\hline
\end{tabular}
\end{tabular}




\section{Discussion}

In this study, about 4 out of 10 parents reported various levels of phone use (meal distraction) during mealtimes. Descriptive analyses revealed more frequent family meals among the group with no phone use compared to the group with phone use during mealtime. Dinner was the most commonly shared family meal for both groups. Parental phone use was associated with less use of positive parental feeding practices such as modelling and family food environment and more use of negative parental feeding practices like emotion regulation and use of pressure to eat. Finally, parental phone use was associated with a lower frequency of daily family breakfast and dinner. Few studies have reported theses associations in this age group (toddlers).

When the child enters toddlerhood around one year of age, it becomes increasingly common to eat the same as the rest of the family and together with their family. This is a challenging period of transition, and in addition both parents increasingly engage in working life again after parental leave, and consequently have less time for other activities (e.g. family meals). We also know from a previous study that adults, including parents of toddlers, are increasingly exposed to distractions from their mobile phones during family mealtime (14). Since parental feeding practices and frequency of family meals are important when it comes to child eating development, this "fight for attention" between the phone and the child, represents a growing dilemma. When parents use their mobile phone in the presence of their children, they are physically present, but distracted and probably unresponsive (25). A small experimental study by Myruski et al. found that infants showed most distress when their mothers were disengaged and used their phones in the presence of their child (25). We found that even in our higher educated sample, as much as $40 \%$ used their phone during meals to some extent. This is higher than reported by an earlier study that found that about $25 \%$ of mothers who participated had been distracted by technology use during mealtime (26). Radesky et al (27) conducted an observational study in a fast-food restaurant assessing young children's behavior in relation to the use of mobile devices by their parents. The parents' use of mobile phones ranged from lying on the dining table to always being occupied with the phone. The study showed that $73 \%$ of the parents used their mobile device while eating, $29 \%$ used their mobile devices during the entire meal, and $15 \%$ looked at their smartphone mobile device while the child was still eating (27).

We found higher frequency of all types of daily shared family meals among the group with no phone use compared to the group with phone use during mealtime. Given that family meals have been reported to influence the eating habits of the child in a positive way (10), these findings are of interest. We found that parental meal distraction (phone use) was associated with lower frequency of daily family breakfast and dinner. It may be that if the parents are conscious about not using their phone during meals, this may be associated with other positive meal habits, such as the importance of having shared family meals. 
Parental meal distraction (e.g. mobile phone use) during meals may influence feeding practices, by less focus on the toddler's food intake, distraction, negative modelling, and use of food as a reward if the child gets impatient. Also, monitoring; i.e. parents keeping track of child's intake of less healthy food might be affected by phone use during meals since the attention is drawn away from the child, i.e. the phone and the child are both "fighting for attention" from the parent. A previous study found that mobile device use among mothers was common and associated with fewer interactions with children during a structured interaction task, particularly nonverbal interactions and that during introduction of an unfamiliar food they had less support and modelling from their mother (28). It is logical to assume that the use of mobile phone during mealtime may influence feeding practices. However, as these are purely associations, opposite directions should be considered, such as parental awareness of demonstrating healthy eating behavior (e.g., modelling) may also influence the use of mobile phone during mealtime.

Strengths and limitations

The cross-sectional data analyzed, provide a snapshot of the study sample and cannot identify any causal associations and the findings may only be generalized to a population of parents with toddlers. The presence of fathers at mealtime should not be neglected, as their presence are reported be to positively associated with less child distractions and more maternal feeding responsiveness (14). In this study we were not able to test statistically for potential differences between mothers and fathers due to the homogeneity of the sample ( $98 \%$ were mothers). Further, by dichotomizing the parents into those who use and do not use mobile phone during mealtime have reduced the extent of variation in data and thus, may increase the risk of bias. Nevertheless, there are several strengths to considered such as the relatively high sample size, the use of well-validated questionnaires and the fact that this study extends previous assumptions and research evidence.

\section{Conclusions}

Mobile phone use is increasingly taking up time and attention and may thereby change the way we behave. This may also impact important parental tasks such as feeding practices and attention towards children during family meals. In the present study, 4 out of 10 parents with toddlers reported various levels of phone use during mealtimes. Parental phone use was associated with less use of positive parental feeding practices, more use of negative parental feeding practices and lower frequency of daily family breakfast and dinner. Our results highlight the importance of making parents aware of meal distractions like mobile phone use. Future studies should include fathers and explore longitudinal data.

\section{Abbreviations}

NCD's

non-communicable diseases

TV

television

Page $11 / 15$ 
CFPQ

Comprehensive Feeding Practices Questionnaire

SES

socio-economic status

OR

Odds Ratio

Cl

Confidence Interval

\section{Declarations}

\section{Ethics approval and consent to participate}

The study was approved by the Norwegian Centre for Research Data, and by the Faculty ethics committee and has been conducted in line with the Helsinki Declaration of 1985, revised 2008. The study was voluntary and the parents consented at the website that they wanted to participate after reading written information about the study.

\section{Consent for publication}

Not applicable.

\section{Availability of data and materials}

The dataset supporting the conclusions of this article will be available in the UiA Open Research repository (https://dataverse.no/dataverse/uia)

\section{Competing interests}

The authors declare that they have no competing interests.

\section{Funding}

This study is funded by the University of Agder. The financial contributor was not involved in designing the study, collection, analyses, and interpretation of data or in writing the manuscript.

\section{Authors' contributions}

FNV, ERH, and NCØ initiated and designed the study. FNV, EG and NCØ initiated and developed the current paper. MR performed the data collection supervised by ERH, FNV, and NCØ. EG performed the statistical analyses of the current paper. FNV, EG and NCØ drafted the first version of the manuscript. All authors 
gave substantial input to the manuscript. All authors contributed to, read, and approved the final version of this manuscript.

\section{Acknowledgment}

The authors wish to thank the participants.

\section{References}

1. Clark H, Coll-Seck AM, Banerjee A, Peterson S, Dalglish SL, Ameratunga S, et al. A future for the world's children? A WHO-UNICEF-Lancet Commission. Lancet. 2020;395(10224):605-58.

2. Mitchell GL, Farrow $C$, Haycraft E, Meyer C. Parental influences on children's eating behaviour and characteristics of successful parent-focussed interventions. Appetite. 2013;60(1):85-94.

3. WHO. Global action plan for the prevention and control of noncommunicable diseases 2013-2020. Switzerland: Geneva; 2013.

4. WHO. Report of the commission on ending childhood obesity. Geneva: World Health Organization; 2016.

5. Schwarzenberg SJ, Georgieff MK. Advocacy for Improving Nutrition in the First 1000 Days to Support Childhood Development and Adult Health. Pediatrics. 2018;141(2).

6. Sælensminde K, Johansson L, Helleve A. Societals gains of adeherence to dietary guidelines (In Norwegian). Oslo: The Norwegian Directorate of Health; 2016.

7. Blissett J, Fogel A. Intrinsic and extrinsic influences on children's acceptance of new foods. Physiol Behav. 2013;121:89-95.

8. Harrison K, Bost KK, McBride BA, Donovan SM, Grigsby-Toussaint DS, Kim J, et al. Toward a Developmental Conceptualization of Contributors to Overweight and Obesity in Childhood: The SixCs Model. Malden, MA:2011. p. 50 - 8.

9. Larson RW, Branscomb KR, Wiley AR. Forms and functions of family mealtimes: multidisciplinary perspectives. New Dir Child Adolesc Dev. 2006(111):1-15.

10. Scaglioni S, De Cosmi V, Ciappolino V, Parazzini F, Brambilla P, Agostoni C. Factors Influencing Children's Eating Behaviours. Nutrients. 2018;10(6).

11. Shloim N, Edelson LR, Martin N, Hetherington MM. Parenting Styles, Feeding Styles, Feeding Practices, and Weight Status in 4-12 Year-Old Children: A Systematic Review of the Literature. Frontiers in psychology. 2015;6:1849.

12. Vaughn AE, Ward DS, Fisher JO, Faith MS, Hughes SO, Kremers SP, et al. Fundamental constructs in food parenting practices: a content map to guide future research. Nutr Rev. 2016;74(2):98-117.

13. Internet World Statistics. Internet Growth Statistics: Miniwatts Marketing Group; 2020 [Available from: https://www.internetworldstats.com/emarketing.htm. 
14. Saltzman JA, Musaad S, Bost KK, McBride BA, Fiese BH. Associations between father availability, mealtime distractions and routines, and maternal feeding responsiveness: An observational study. $J$ Fam Psychol. 2019;33(4):465-75.

15. Fiese BH, Jones BL, Jarick JM. Family mealtime dynamics and food consumption: An experimental approach to understanding distractions. Couple Family Psychology: Research Practice. 2015;4(4):199-211.

16. Kong A, Jones BL, Fiese BH, Schiffer LA, Odoms-Young A, Kim Y, et al. Parent-child mealtime interactions in racially/ethnically diverse families with preschool-age children. Eat Behav. 2013;14(4):451-5.

17. Malhotra K, Herman AN, Wright G, Bruton Y, Fisher JO, Whitaker RC. Perceived benefits and challenges for low-income mothers of having family meals with preschool-aged children: childhood memories matter. Journal of the Academy of Nutrition Dietetics. 2013;113(11):1484-93.

18. Powell EM, Frankel LA, Umemura T, Hazen N. The relationship between adult attachment orientation and child self-regulation in eating: The mediating role of persuasive-controlling feeding practices. Eat Behav. 2017;26:121-8.

19. Ventura AK, Teitelbaum S. Maternal Distraction During Breast- and Bottle Feeding Among WIC and non-WIC Mothers. J Nutr Educ Behav. 2017;49(7 Suppl 2):169-S76. :.e1..

20. Verhage CL, Gillebaart M, van der Veek SMC, Vereijken C. The relation between family meals and health of infants and toddlers: A review. Appetite. 2018;127:97-109.

21. Hammons AJ, Fiese BH. Is frequency of shared family meals related to the nutritional health of children and adolescents? Pediatrics. 2011;127(6):e1565-74.

22. Røed M, Hillesund ER, Vik FN, Van Lippevelde W, Øverby NC. The Food4toddlers study - study protocol for a web-based intervention to promote healthy diets for toddlers: a randomized controlled trial. BMC Public Health. 2019;19(1):563.

23. Musher-Eizenman D, Holub S. Comprehensive Feeding Practices Questionnaire: validation of a new measure of parental feeding practices. J Pediatr Psychol. 2007;32(8):960-72.

24. Melbye EL, Øverby NC, Øgaard T. Child consumption of fruit and vegetables: the roles of child cognitions and parental feeding practices. Public Health Nutr. 2012;15(6):1047-55.

25. Myruski S, Gulyayeva O, Birk S, Pérez-Edgar K, Buss KA, Dennis-Tiwary TA. Digital disruption? Maternal mobile device use is related to infant social-emotional functioning. Dev Sci. 2018;21(4):e12610.

26. McDaniel BT, Coyne SM. "Technoference": The interference of technology in couple relationships and implications for women's personal and relational well-being. Psychology of Popular Media Culture. 2016;5(1):85-98.

27. Radesky JS, Kistin CJ, Zuckerman B, Nitzberg K, Gross J, Kaplan-Sanoff M, et al. Patterns of mobile device use by caregivers and children during meals in fast food restaurants. Pediatrics. 2014;133(4):e843-9. 
28. Radesky J, Miller AL, Rosenblum KL, Appugliese D, Kaciroti N, Lumeng JC. Maternal mobile device use during a structured parent-child interaction task. Academic pediatrics. 2015;15(2):238-44.

\section{Supplementary Files}

This is a list of supplementary files associated with this preprint. Click to download.

- Additionalfile1.docx 